\title{
EDITORIAL
}

\section{Anterior capsulotomy and deep brain stimulation}

\author{
Emad N. Eskandar, MD \\ Department of Neurosurgery, Massachusetts General Hospital, Boston, Massachusetts
}

I $\mathrm{N}$ this paper, the authors performed a literature review and compared the results of anterior capsulotomy (AC) to those of deep brain stimulation (DBS) of the ventral capsule/ventral striatum for the treatment of obsessivecompulsive disorder (OCD). ${ }^{1}$ Capsulotomy has been in use for decades, whereas DBS for OCD is relatively new. The paper used the standard criteria for improvement, a YaleBrown Obsessive-Compulsive Scale (YBOCS) score reduction of $35 \%$ or greater, and a YBOCS score of less than 8 for remission. The results demonstrate that the techniques are relatively comparable, with AC on average demonstrating a slightly greater effect compared to DBS. The authors argue that the adoption of DBS has more to do with patient and clinician preference than with greater clinical efficacy.

There are some limitations to the study that are worth noting. Virtually all of the papers included in the review were retrospective and open-label; hence the possibility of unintended bias exists. The papers in the review are not contemporaneous, with AC having the benefit of a much longer time to evolve. In addition, there are other ablative procedures, such as cingulotomy, that are not considered in the paper.

Nonetheless, the authors make an important point: specifically, that based on the available literature, ablative procedures may confer a clinical benefit similar to DBS. Moreover, ablative procedures are generally faster, more cost-effective, and require considerably less postoperative management, all of which are relevant factors given the current downward pressure on health care costs. Conversely, psychiatric neurosurgery has a checkered history in the popular mind, and the use of DBS, which is adjustable and reversible, makes it philosophically and ethically less challenging for providers and patients alike. Thus the use of DBS could lead more patients with medically intractable disease to benefit from surgical therapy. Ideally, there would be prospective randomized controlled trials to answer these questions definitively, but for the moment the controversy will continue.

http://thejns.org/doi/abs/10.3171/2014.9.JNS14925

\section{Reference}

1. Pepper J, Hariz M, Zrinzo L: Deep brain stimulation versus anterior capsulotomy for obsessive-compulsive disorder: a review of the literature. J Neurosurg [epub ahead of print January 30, 2015. DOI: 10.3171/2014.11.JNS132618]

\section{Response}

\section{Joshua Pepper, MD, ${ }^{1}$ Marwan Hariz, MD, PhD, ${ }^{1,2}$ and Ludvic Zrinzo, MD, PhD ${ }^{1}$}

1 Unit of Functional Neurosurgery, Sobell Department of Motor Neuroscience and Movement Disorders, University College London Institute of Neurology, Queen Square, London; and 'Department of Neurosurgery, Umeå University, Umeå, Sweden

We appreciate the opportunity to expand on the topics raised in the Editorial by Dr. Eskandar. We agree that "capsulotomy has been in use for decades, whereas DBS for OCD is relatively new." However, our literature review was restricted to papers using the YBOCS scale as an outcome measure, disregarding older papers published before its introduction in $1989 .{ }^{1}$ As a result, included papers on $\mathrm{AC}$ and DBS were from a similar chronological period (published between 2002 and 2013, and between 2005 and 2012, respectively). Therefore, we cannot concur with the Editorial statement, "The papers in the review are not contemporaneous."

Of course, we are aware of the limitations of such a review. "Virtually all of the papers included in the review 
were retrospective and open-label; hence the possibility of unintended bias exists." Indeed, we attempted to reduce the bias introduced by patient selection by stratifying outcome according to the severity of initial patient symptoms.

It was not our intention to consider "other ablative procedures, such as cingulotomy." The aim was to compare two surgical procedures targeting roughly the same anatomical brain region; specifically, the anterior limb of the internal capsule and adjacent structures. Therefore, we did not include cingulotomy, subcaudate tractotomy, or limbic leukotomy; nor did we consider papers on DBS for OCD in which other brain regions, such as the subthalamic nucleus, the bed nucleus of the stria terminalis, the inferior thalamic peduncle, and the medial zona incerta were targeted. ${ }^{2}$

The author states that "psychiatric neurosurgery has a checkered history in the popular mind, and the use of DBS, which is adjustable and reversible, makes it philosophically and ethically less challenging for providers and patients alike." Although we agree with this statement, we find it unjust to amalgamate and confound stereotactic ablative neurosurgery for OCD (and other psychiatric illnesses) with the nonstereotactic lobotomy. Our findings suggest that stereotactic ablative surgery is worthy of consideration on its own merits with respect to both efficacy and safety. Hence our conclusion that "the adoption of DBS has more to do with patient and clinician preference than with greater clinical efficacy."

We agree that "the use of DBS could lead more patients with medically intractable disease to benefit from surgical therapy." However, a more expensive, high-maintenance, nonablative procedure should only retain its appeal if it matches or supersedes the efficacy and safety of an ablative approach. It is worth noting that despite 15 years of intense academic activity since the introduction of DBS for OCD in 1999, ${ }^{4}$ the number of published patients who received DBS for OCD is limited to approximately 100 worldwide. ${ }^{3}$ Despite the "reversibility" and "better social acceptance" of DBS, the major obstacle remains the dif- ficulty in recruiting patients for surgical trials, including DBS trials. Indeed, it is still acknowledged by those active in the field that "DBS remains an experimental treatment for medication refractory OCD." 3

Again, we agree that "ideally, there would be prospective randomized controlled trials to answer these questions definitively, but for the moment, the controversy will continue." In the meantime, our analysis of the relevant literature is a modest step toward addressing that controversy.

The deeply negative legacy of widely destructive nonstereotactic psychosurgery taints the promise of ablative stereotactic surgery in mental disorders. With our review, we hope to further dissociate stereotactic ablative neurosurgery from lobotomy by comparing its results with those of DBS at a similar brain region. The baby should not be thrown away with the bath water.

In summary, we hope that our literature review of AC versus DBS for OCD will challenge unfounded concepts based on prejudice alone and unsupported by the scientific literature. Perhaps it may even stimulate philosophical and ethical debate on the various merits of stereotactic ablative surgery and DBS for OCD and other mental disorders.

\section{References}

1. Goodman WK, Price LH, Rasmussen SA, Mazure C, Fleischmann RL, Hill CL, et al: The Yale-Brown Obsessive Compulsive Scale. I. Development, use, and reliability. Arch Gen Psychiatry 46:1006-1011, 1989

2. Krack P, Hariz MI, Baunez C, Guridi J, Obeso JA: Deep brain stimulation: from neurology to psychiatry? Trends Neurosci 33:474-484, 2010

3. Morishita T, Fayad SM, Goodman WK, Foote KD, Chen D, Peace DA, et al: Surgical neuroanatomy and programming in deep brain stimulation for obsessive compulsive disorder. Neuromodulation 17:312-319, 2014

4. Nuttin B, Cosyns P, Demeulemeester H, Gybels J, Meyerson B: Electrical stimulation in anterior limbs of internal capsules in patients with obsessive-compulsive disorder. Lancet 354:1526, 1999 